\title{
Positional Match Demands of Professional Rugby League Competition
}

\author{
Anita C. Sirotic, ${ }^{1,2}$ Hayden Knowles, ${ }^{2}$ Craig Catterick, ${ }^{2}$ and Aaron J. Coutts ${ }^{1}$ \\ ${ }^{1}$ Human Performance Laboratory, School of Leisure, Sport and Tourism, University of Technology, Sydney, Australia; and \\ ${ }^{2}$ High Performance Department, Parramatta Eels Rugby League Football Club, Parramatta, Australia
}

\begin{abstract}
Sirotic, AC, Knowles, H, Catterick, C, and Coutts, AJ. Positional match demands of professional rugby league competition. J Strength Cond Res 25(11): 3076-3087, 2011-The purpose of this study was to examine the differences in physical performance and game-specific skill demands between 5 positional groups in a professional rugby league team. Positional groups consisted of the backs $(n=8)$, forwards $(n=8)$, fullback $(n=7)$, hooker $(n=8)$, and service players $(n=8)$. Time-motion analysis was used to determine physical performance measures (exercise intensity, distance travelled, time, frequency, and speed measures) and game-specific skill measures (ball carries, supports, ball touches, play the balls, and tackling indices) per minute of playing time. The main finding was that the fullback completed more very high-intensity running (VHIR) because of more support runs when compared to all other positional groups $(p=0.017)$. THe VHIR $(p=0.004)$ and sprinting indices $(p<0.002)$ were also greater in the second half of a match for the fullback than in any other positional group. The hooker spent more time jogging than the backs and forwards $(p<0.001)$ and touched the ball on more occasions than any other positional group $(p<0.001)$. The backs spent more time walking than the forwards, hooker, and service players $(p<0.001)$. The forwards, hooker, and service players completed more tackles per minute during a match than the backs and fullback $(p<0.001)$. The fullback and forwards also ran the ball on more occasions than the backs, hooker, and service players did $(p<0.001)$. These results show that positional roles play an important part in determining the amount of physical and game-specific skill involvement during match play.
\end{abstract}

KEY WoRDS match analysis, high-intensity activity, gamespecific skill

Address correspondence to Dr. Aaron J. Coutts, aaron.coutts@uts.edu.au. 25(11)/3076-3087

Journal of Strength and Conditioning Research

(C) 2011 National Strength and Conditioning Association

3076 Journal of Strength and Conditioning Research

\section{INTRODUCTION}

$\mathrm{T}$ ime-motion analysis is a noninvasive method of quantifying the physical and game-specific movement patterns of team sports. Recently, timemotion analysis has been used to determine the physical demands of different positional roles in a range of professional team sports, including soccer $(16,18,19)$, Australian Football League $(2,4)$, rugby union $(5,7)$, and field hockey (22). However, there is relatively little information regarding the physical and game-specific skill demands of professional rugby league $(12,21,23)$. The information gained from these analyses can be used to assist coaches in developing position-specific training programs and to direct specific training activities depending on a player's positional demands.

Previous time-motion studies have examined the physical and technical demands of rugby league using 2-4 general positional groups within a team $(12,14,15,23)$. Two of these earlier studies reported differences in both physical movement and technical skill involvement between forwards and backs during match play at the elite level $(14,15)$. However, these previous studies did not provide a detailed analysis on the demands of several specific playing positions such as the hooker, fullback, and service players (i.e., half-back, fiveeighth and lock). Therefore, there is a need for further detailed match analysis to describe the physical and gamespecific skill demands of these playing positions in professional rugby league.

Rule changes may also have changed the physical and game-specific skill requirements of professional rugby league players (12). Indeed, results from previous studies suggest that rule changes (i.e., the introduction of the $10-\mathrm{m}$ rule or the limited interchange rule) have affected the physical movement demands of rugby league players, altering the distances covered and time spent at high-intensities $(12,14,15,23)$. However, since the reintroduction of the limited interchange rule in 2001, only 2 studies have investigated the physical demands of positional roles in professional rugby league, and none have examined their skill demands. Accordingly, there is a clear need to gain a better understanding of the positionspecific skill and physical demands of professional rugby league players. Such information may be used by coaches to develop position-specific training drills that can be then used 
as part of a comprehensive training program to prepare for both the physical and skill demands of their playing position. Therefore, the purpose of this study was to analyze the physical and game-specific skill match demands of 5 positional groups (i.e., backs, forwards, fullback, hooker, and service players) during professional rugby league match play.

\section{MethodS}

\section{Experimental Approach to the Problem}

In this study, time-motion and notational analysis was used to investigate the movement and game-specific skill demands of top-level rugby league players from various playing positions. The study also examined the effect of physical activity completed during the first half on the game demands during the second half.

\section{Subjects}

Video analysis was performed on 17 professional (mean $\pm S D$; age $=24.8 \pm 3.1$ years, body mass $=95.7 \pm 8.1 \mathrm{~kg}$, and stature $=1.84 \pm 0.05 \mathrm{~m}$ ) rugby league players during 16 official National Rugby League (NRL) matches in Australia. The NRL is arguably the leading rugby league competition in the world. All players were full-time professional players and were contracted to the same NRL club (Parramatta Eels). All players were video monitored at the club's home ground (Parramatta Stadium Parramatta, Australia) over 2 seasons. All matches were played during the March to September period and under the same playing rules. Although environmental conditions were not controlled, games that were played in wet conditions were not analyzed. During the period of analysis, the team won $52.1 \%$ of games. Five positional groups were analyzed: (a) Backs: winger and center $(n=8$; mean $\pm S D$; age $=23.7 \pm 2.2$ years, body mass $=92.9 \pm 5.5 \mathrm{~kg}$, stature $=$ $1.82 \pm 0.04 \mathrm{~m})$; (b) Forwards: prop and second row $(n=8$; mean $\pm S D$; age $=27.8 \pm 3.0$ years, body mass $=106.0 \pm 3.2$ $\mathrm{kg}$, stature $=1.86 \pm 0.03 \mathrm{~m})$; (c) Fullback: $(n=7$; mean $\pm S D$; age $=23.8 \pm 0.4$ years, body mass $=87.8 \pm 0.4 \mathrm{~kg}$, stature $=$ $1.81 \pm 0.04 \mathrm{~m}) ;(\mathrm{d})$ Hooker. $(n=8$; mean $\pm S D$; age $=24.3 \pm 1.2$ years, body mass $=91.7 \pm 7.6 \mathrm{~kg}$, stature $=1.79 \pm 0.04 \mathrm{~m})$; and (d) Service players: half-back, five-eighth and lock $(n=8$; mean $\pm S D$; age $=24.8 \pm 3.7$ years, body mass $=92.3 \pm 2.9 \mathrm{~kg}$, stature $=1.82 \pm 0.03 \mathrm{~m}$ ).

The positional groups were chosen in consultation with professional coaching staff during the observation of previous NRL matches. The fullback and hooker were analyzed separately, because of their unique tactical roles. Prior intention to videorecord a playing position was not made known to any player, and players were randomly chosen by the video camera operator. Because of the limited number of players in each position within the club, individuals were analyzed 1-6 times. Informed consent was voluntarily obtained from all players before the commencement of the study. Ethical approval was granted by the University Human Research Ethics Committee.

\section{Procedures}

Video Procedures. The video procedures used in this study have been described $(8,21)$. Video monitoring was recorded using standard video cameras (Panasonic NV-DX100, Osaka, Japan, and JVC GR-DVL 9800, Yokohama, Japan, digital video cameras) by a trained video camera operator. During videorecording, the player who started in the position to be monitored was videotaped until this player left the ground through substitution. The camera remained filming this player on the interchange bench until the player returned to the field. If a selected player being video monitored was replaced because of injury, sent from the field for rule indiscretion, or changed playing position, then that player's match was not analyzed.

Physical Match Analysis. Physical match analysis was performed by a trained operator, using a computer-based tracking (CBT) system (Trak Performance version 2.2, SportsTec Pty Ltd., Sydney, Australia) according to previous methods (21). Analysis of physical match activities was determined using the following movement categories based on previous time-motion data (18): Standing (0-1.0 $\left.\mathrm{km} \cdot \mathrm{h}^{-1}\right)$; Walking (1.1-7.0 $\left.\mathrm{km} \cdot \mathrm{h}^{-1}\right)$; Jogging (7.1-13.0 $\mathrm{km} \cdot \mathrm{h}^{-1}$ ); Low-intensity running (LIR; running speed $<$ $13.1 \mathrm{~km} \cdot \mathrm{h}^{-1}$ ); High-intensity running (HIR; running speed $>13.1 \mathrm{~km} \cdot \mathrm{h}^{-1}$ ); Very high-intensity running (VHIR; running speed $\left.>18.6 \cdot \mathrm{km} \mathrm{h}^{-1}\right)$; Sprinting $\left(>24.0 \mathrm{~km} \cdot \mathrm{h}^{-1}\right)$; and Backward movement.

Match Performance Measures. The relative time, intensity of exercise, mean duration and distance, and frequency of each movement were calculated and totaled for the first half, second half, and match. Additionally, the mean times between sprints and mean playing speed were also determined. Changes in speed were calculated according to the methods of Sirotic et al. (21). High-Intensity to low-intensity ratio was calculated according to methods of Coutts et al. (3). Because of player interchange, measures of exercise intensity, frequency, and changes in speed were expressed per minute of playing time.

Effect of First-Half Activity on the Second Half. The effect of first-half physical activity on second-half physical performance was examined according to previous methods (18). The median split technique was used to divide NRL match data $(n=39)$ for first-half total intensity of exercise into either a high group (i.e., total intensity of exercise was above the median score) or low group (i.e., total intensity of exercise was below the median score). Analyses were then performed to investigate whether high or low total exercise intensity during the first half affected the total intensity of exercise during the second half. The same procedure was also performed on first-half HIR exercise data.

Game-Specific Skill Match Analysis Data. Game-specific skill data from each positional group $(n=39)$ were manually coded by a trained operator according to the previous 
TABLE 1. Differences in match work rate and distance measures between the 5 positional groups $(n=39$; mean $\pm S D) .{ }^{*}$

\begin{tabular}{|c|c|c|c|c|c|c|c|c|}
\hline Movement & Variable & $\begin{array}{c}\text { Backs } \\
(n=8)\end{array}$ & $\begin{array}{l}\text { Forwards } \\
(n=8)\end{array}$ & $\begin{array}{c}\text { Fullback } \\
(n=7)\end{array}$ & $\begin{array}{l}\text { Hooker } \\
(n=8)\end{array}$ & $\begin{array}{l}\text { Service } \\
(n=8)\end{array}$ & $\begin{array}{c}\text { TEM } \\
95 \% \mathrm{Cl}\end{array}$ & $\begin{array}{c}\text { Main effect } \\
p \text { value }\end{array}$ \\
\hline \multirow[t]{2}{*}{ Backward } & Total work-rate $\left(\mathrm{m} \cdot \mathrm{min}^{-1}\right)$ & $100.4 \pm 6.7$ & $105.4 \pm 10.7$ & $110.3 \pm 9.5$ & $110.1 \pm 9.2$ & $105.3 \pm 9.0$ & $2.2-4.3$ & 0.261 \\
\hline & Work-rate $\left(\mathrm{m} \mathrm{min}^{-1}\right)$ & $6.5 \pm 2.2 \dagger$ & $5.3 \pm 1.1 \dagger$ & $19.1 \pm 4.2 \ddagger \S \|$ | & $4.6 \pm 2.0 \dagger$ & $7.2 \pm 2.0 \dagger$ & $0.8-1.7$ & $<0.001 \#$ \\
\hline \multirow[t]{4}{*}{ Sprinting } & Mean distance $(\mathrm{m})$ & $3.9 \pm 0.3 \dagger$ & $3.6 \pm 0.4 \dagger$ & $5.4 \pm 0.4 \uparrow \S \| \uparrow$ & $3.7 \pm 0.4 \dagger$ & $3.8 \pm 0.3 \dagger$ & $0.8-1.5$ & $<0.001 \#$ \\
\hline & Work rate $\left(\mathrm{m} \cdot \mathrm{min}^{-1}\right)$ & $5.4 \pm 1.8$ & $4.7 \pm 1.3$ & $7.1 \pm 3.0 \| 9$ & $3.5 \pm 2.0 \dagger$ & $3.9 \pm 0.9 \dagger$ & $0.4-0.9$ & $0.008 \#$ \\
\hline & Mean distance $(\mathrm{m})$ & $20.1 \pm 3.7$ & $19.1 \pm 4.9$ & $16.0 \pm 1.7$ & $16.8 \pm 1.6$ & $17.9 \pm 3.5$ & $0.9-1.9$ & 0.108 \\
\hline & Maximum distance $(\mathrm{m})$ & $48.9 \pm 11.0$ & $44.9 \pm 14.6$ & $41.9 \pm 9.2$ & $36.2 \pm 10.0$ & $43.5 \pm 9.0$ & $3.0-6.0$ & 0.141 \\
\hline \multirow[t]{2}{*}{ VHIR } & Work-rate $\left(\operatorname{mnnmin}^{-1}\right)$ & $12.7 \pm 2.9$ & $13.3 \pm 1.6$ & $17.5 \pm 4.6 \| \Phi$ & $11.3 \pm 5.8 \dagger$ & $11.2 \pm 2.3 \dagger$ & $0.9-1.8$ & $0.017 \#$ \\
\hline & Mean distance $(\mathrm{m})$ & $12.0 \pm 0.8$ & $11.5 \pm 1.2$ & $10.7 \pm 0.5$ & $10.9 \pm 0.7$ & $11.1 \pm 1.1$ & $0.6-1.2$ & 0.061 \\
\hline \multirow[t]{2}{*}{ HIR } & Work rate $\left(\mathrm{m} \cdot \mathrm{min}^{-1}\right)$ & $29.6 \pm 5.6$ & $36.9 \pm 6.6$ & $37.5 \pm 7.2$ & $35.2 \pm 11.8$ & $32.9 \pm 5.8$ & $1.9-3.8$ & 0.269 \\
\hline & Mean distance $(\mathrm{m})$ & $9.2 \pm 0.5 \dagger \|$ & $9.1 \pm 0.2$ & $8.6 \pm 0.3 \neq$ & $8.6 \pm 0.3 \ddagger$ & $8.6 \pm 0.4$ & $0.3-0.5$ & $0.003 \#$ \\
\hline \multirow[t]{2}{*}{ LIR } & Work rate $\left(\mathrm{m} \cdot \min ^{-1}\right)$ & $70.8 \pm 5.9$ & $68.6 \pm 4.7$ & $72.8 \pm 3.1$ & $73.9 \pm 5.2$ & $72.4 \pm 4.9$ & $1.4-2.9$ & 0.249 \\
\hline & Mean distance $(\mathrm{m})$ & $6.3 \pm 0.6$ & $5.7 \pm 0.2$ & $6.0 \pm 0.3$ & $5.7 \pm 0.5$ & $5.9 \pm 0.4$ & $0.2-0.4$ & 0.052 \\
\hline \multirow[t]{2}{*}{ Jogging } & Work rate $\left(\mathrm{m} \cdot \mathrm{min}^{-1}\right)$ & $36.3 \pm 3.6 \| \uparrow$ & $40.3 \pm 5.0 \|$ & $40.2 \pm 3.3 \|$ & $47.2 \pm 2.5 \ddagger \S \dagger$ & $44.1 \pm 5.0 \ddagger$ & $1.5-3.0$ & $<0.001 \#$ \\
\hline & Mean distance $(\mathrm{m})$ & $6.6 \pm 0.3 \|$ & $6.7 \pm 0.2$ & $6.7 \pm 0.3$ & $7.3 \pm 0.5 \ddagger$ & $7.2 \pm 0.5$ & $0.3-0.5$ & $0.004 \#$ \\
\hline \multirow[t]{2}{*}{ Walking } & Work rate $\left(\mathrm{m} \cdot \mathrm{min}^{-1}\right)$ & $34.6 \pm 3.8 \S \| 9$ & $28.3 \pm 2.3 \neq$ & $32.6 \pm 1.3 \|$ & $26.7 \pm 3.9 \ddagger$ & $28.3 \pm 2.5 \neq \dagger$ & $0.8-1.7$ & $<0.001 \#$ \\
\hline & Mean distance $(\mathrm{m})$ & $5.9 \pm 0.8 \S \| \Phi$ & $4.7 \pm 0.5 \ddagger$ & $5.4 \pm 0.4 \|$ & $4.2 \pm 0.5 \ddagger$ & $4.7 \pm 0.6+\dagger$ & $0.2-0.5$ & $<0.001 \#$ \\
\hline
\end{tabular}

${ }^{*} \mathrm{VHIR}=$ very high-intensity running; HIR = high-intensity running; TEM $=$ technical error of measurement; $\mathrm{Cl}=$ confidence interval; ANOVA $=$ analysis of variance.

$\dagger$ Significantly different from fullback $(p<0.05)$ and difference above the upper level of TEM $95 \% \mathrm{Cl}$.

$\ddagger$ Significantly different from backs (Bonferroni Post hoc; $p<0.05$ ) and difference above the upper level of TEM $95 \% \mathrm{CI}$.

$\S$ Significantly different from forwards $(p<0.05)$ and difference above the upper level of TEM $95 \% \mathrm{Cl}$.

$\|$ Significantly different from hooker $(p<0.05)$ and difference above the upper level of TEM $95 \% \mathrm{Cl}$.

I Significantly different from service players $(p<0.05)$ and difference above the upper level of TEM 95\% Cl.

\#One-way ANOVA significant main effect $(p<0.05)$. 


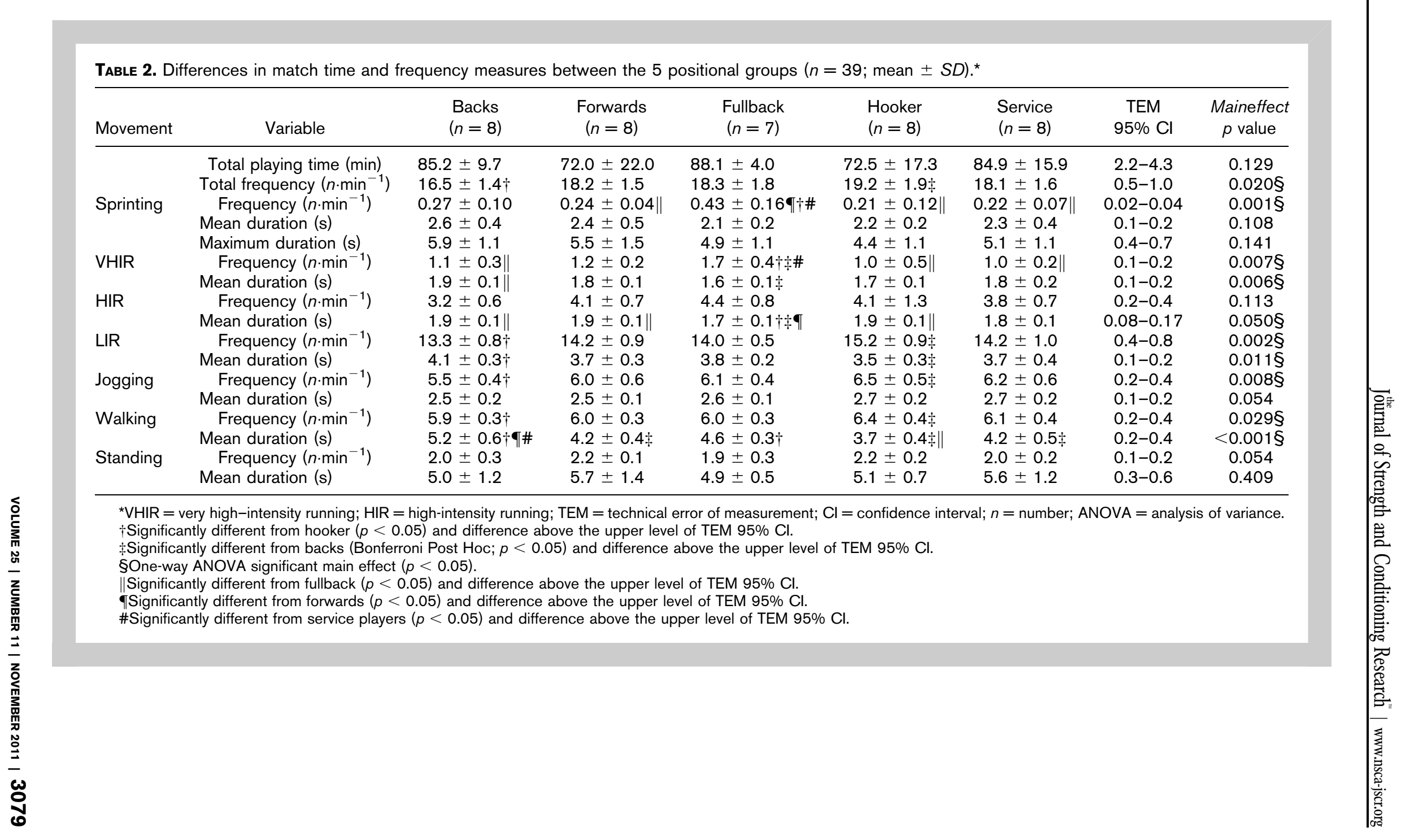



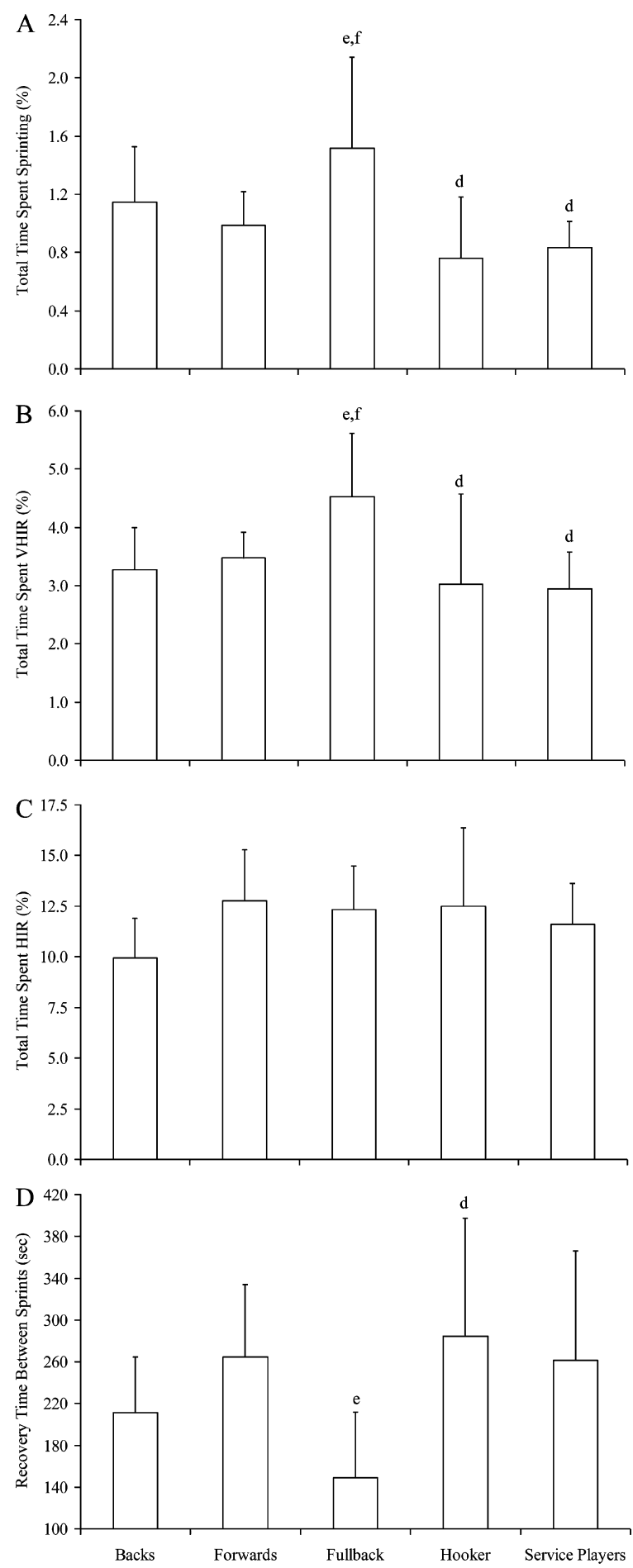

Figure 1. Relative time spent (A) sprinting, (B) very high-intensity running (VHIR), and (C) high-intensity running (HIR), and mean recovery time between (D) sprinting for the 5 positional groups during rugby league match play $(n=39)$. ${ }^{b}$ Significantly different from backs $(p<0.01)$ and difference above the upper level of TEM $95 \%$ confidence interval $(\mathrm{Cl})$. ${ }^{\circ}$ Significantly different from forwards $(p<0.01)$ and difference above the upper level of TEM $95 \% \mathrm{Cl}$. dSignificantly different from fullback $(p<0.03)$ and difference above the upper level of TEM $95 \% \mathrm{Cl}$. eSignificantly different from hooker $(p<0.03)$ and difference above the upper level of TEM $95 \% \mathrm{Cl}$. 'Significantly different from service players $(p<0.01)$ and difference above the upper level of TEM $95 \% \mathrm{Cl}(14)$. methods (21). The number of involvements for offensive and defensive skills were determined per minute of playing time for the first half, second half, and match.

Offensive Skill Measures. Offensive game-specific skills were defined according to the previous methods (21) and included the following: Ball carry: A player in possession of the ball traveled a distance of $>2$ steps in any direction. Support run: An offensive movement performed close to a ball runner with the intention of receiving the ball or drawing a defender away from the ball runner in an effort to promote the ball toward the opposition's goal line. Touch of the ball: A single period of time during which a player was in contact with the ball before the ball is released. Play the ball: A tackled player rose to his feet and played the ball backward with a striking action.

Defensive Skill Measures. Defensive game-specific skills were defined according to previous methods (21) and included the following: Tackle: A player engaged in physical contact with an opponent in an effort to stop the opponent from promoting the ball toward the player's goal line. Tackle (one on one): A tackle was made by one player without the assistance of any other player. Tackle win: The effectiveness of a tackle to allow a defensive player involved in the tackle to be on-side before an opponent has played the ball and usually involves a high level of physical exertion. If the defensive player returned to an onside position simultaneously when the ball was played, this was regarded as a neutral tackle and was not 
TABLE 3. Differences in work-rate and frequency measures in the first half and second half between the 5 positional groups $(n=39 ;$ mean $\pm S D) .{ }^{*}$

\begin{tabular}{|c|c|c|c|c|c|c|c|c|}
\hline Variable & Half & $\begin{array}{l}\text { Backs } \\
(n=8)\end{array}$ & $\begin{array}{l}\text { Forwards } \\
(n=8)\end{array}$ & $\begin{array}{c}\text { Fullback } \\
(n=7)\end{array}$ & $\begin{array}{l}\text { Hooker } \\
(n=8)\end{array}$ & $\begin{array}{l}\text { Service } \\
(n=8)\end{array}$ & $\begin{array}{c}\text { TEM } \\
95 \% \mathrm{Cl}\end{array}$ & $\begin{array}{c}\text { Main effect } \\
p \text { value }\end{array}$ \\
\hline \multirow{2}{*}{$\begin{array}{l}\text { Playing time } \\
\text { (min) }\end{array}$} & First & $43.8 \pm 3.0$ & $35.5 \pm 11.0$ & $43.6 \pm 2.6$ & $35.8 \pm 13.7$ & $42.5 \pm 6.7$ & $2.2-4.3$ & 0.124 \\
\hline & Second & $41.4 \pm 7.9$ & $36.5 \pm 11.7$ & $44.4 \pm 2.6$ & $36.8 \pm 10.9$ & $42.4 \pm 9.8$ & $2.2-4.3$ & 0.121 \\
\hline \multirow{2}{*}{$\begin{array}{l}\text { Sprinting work rate } \\
\left(\mathrm{m} \cdot \mathrm{min}^{-1}\right)\end{array}$} & First & $6.1 \pm 2.4$ & $6.0 \pm 2.3$ & $6.6 \pm 2.6$ & $4.0 \pm 2.4$ & $4.8 \pm 1.4$ & $0.4-0.9$ & 0.175 \\
\hline & Second & $4.7 \pm 1.8$ & $3.6 \pm 1.3 \dagger$ & $7.6 \pm 3.5 \ddagger \S \|$ & $3.6 \pm 2.5 \dagger$ & $3.0 \pm 1.2 \dagger$ & $0.4-0.9$ & $0.002 \Phi$ \\
\hline \multirow{2}{*}{$\begin{array}{l}\text { Time spent sprinting } \\
(\%)\end{array}$} & First & $1.3 \pm 0.5$ & $1.3 \pm 0.4$ & $1.4 \pm 0.6$ & $0.9 \pm 0.5$ & $1.0 \pm 0.3$ & $0.1-0.2$ & 0.198 \\
\hline & Second & $1.0 \pm 0.4$ & $0.8 \pm 0.2 \dagger$ & $1.6 \pm 0.7 \ddagger \S \|$ & $0.8 \pm 0.5 \dagger$ & $0.7 \pm 0.2 \dagger$ & $0.1-0.2$ & $0.002 \Phi$ \\
\hline \multirow{2}{*}{$\begin{array}{l}\text { Sprinting frequency } \\
\left(n \cdot \min ^{-1}\right)\end{array}$} & First & $0.3 \pm 0.1$ & $0.3 \pm 0.1 \#$ & $0.4 \pm 0.2$ & $0.3 \pm 0.2$ & $0.3 \pm 0.1$ & $0.0-0.04$ & 0.084 \\
\hline & Second & $0.2 \pm 0.1 \dagger$ & $0.2 \pm 0.1 \dagger \#$ & $0.5 \pm 0.2 \ddagger \S \|^{\star \star}$ & $0.2 \pm 0.2 \dagger$ & $0.2 \pm 0.1 \dagger$ & $0.0-0.04$ & 0.0019 \\
\hline \multirow{2}{*}{$\begin{array}{l}\text { VHIR work rate } \\
\left(\mathrm{m} \cdot \mathrm{min}^{-1}\right)\end{array}$} & First & $14.0 \pm 4.3$ & $15.4 \pm 3.0$ & $16.9 \pm 4.1$ & $14.0 \pm 8.7$ & $12.0 \pm 3.1$ & $0.9 t-1.8$ & 0.435 \\
\hline & Second & $11.4 \pm 2.4 \dagger$ & $11.4 \pm 1.4 \dagger$ & $18.1 \pm 5.5 \ddagger \S \|^{\star \star}$ & $10.5 \pm 6.6 \dagger$ & $10.3 \pm 2.2 \dagger$ & $0.9 \mathrm{t}-1.8$ & 0.0049 \\
\hline \multirow{2}{*}{$\begin{array}{l}\text { Time spent at VHIR } \\
\text { (\%) }\end{array}$} & First & $3.6 \pm 1.1$ & $4.0 \pm 0.7$ & $4.4 \pm 1.0$ & $3.8 \pm 2.4$ & $3.1 \pm 0.9$ & $0.2-0.5$ & 0.460 \\
\hline & Second & $3.0 \pm 0.6 \dagger$ & $3.1 \pm 0.5$ & $4.7 \pm 1.3 \S \|^{\star \star}$ & $2.8 \pm 1.7 \dagger$ & $2.8 \pm 0.5 \dagger$ & $0.2-0.5$ & 0.0079 \\
\hline \multirow{2}{*}{$\begin{array}{l}\text { VHIR frequency } \\
\qquad\left(n \cdot \min ^{-1}\right)\end{array}$} & First & $1.2 \pm 0.4$ & $1.3 \pm 0.3$ & $1.6 \pm 0.4$ & $1.3 \pm 0.9$ & $1.0 \pm 0.3$ & $0.1-0.2$ & 0.212 \\
\hline & Second & $1.0 \pm 0.3 \dagger$ & $1.1 \pm 0.2 \dagger$ & $1.7 \pm 0.5 \ddagger \S \|^{\star \star}$ & $1.0 \pm 0.6 \dagger$ & $1.0 \pm 0.3 \dagger$ & $0.1-0.2$ & 0.0039 \\
\hline \multirow{2}{*}{$\begin{array}{l}\text { Rapid acceleration } \\
\quad\left(n \cdot \min ^{-1}\right)\end{array}$} & First & $0.4 \pm 0.2$ & $0.6 \pm 0.1 \#$ & $0.8 \pm 0.3 \|$ & $0.5 \pm 0.4$ & $0.4 \pm 0.1 \dagger$ & $0.1-0.2$ & 0.0409 \\
\hline & Second & $0.4 \pm 0.2 \dagger$ & $0.4 \pm 0.1 \#$ & $0.7 \pm 0.3 \|^{* *}$ & $0.5 \pm 0.3$ & $0.4 \pm 0.1 \dagger$ & $0.1-0.2$ & $0.020 \uparrow$ \\
\hline \multirow{2}{*}{$\begin{array}{l}\text { Rapid decelerations } \\
\quad\left(n \cdot \min ^{-1}\right)\end{array}$} & First & $0.5 \pm 0.1 \#$ & $0.6 \pm 0.2 \#$ & $0.7 \pm 0.2$ & $0.5 \pm 0.2$ & $0.4 \pm 0.1$ & $0.05-0.09$ & 0.0359 \\
\hline & Second & $0.3 \pm 0.1 \dagger \#$ & $0.3 \pm 0.1 \dagger \#$ & $0.7 \pm 0.3 \ddagger \S \|^{* *}$ & $0.3 \pm 0.2 \dagger$ & $0.3 \pm 0.1 \dagger$ & 0.05 to 0.09 & $<0.0019$ \\
\hline
\end{tabular}

*VHIR = very high-intensity running; $\mathrm{HIR}=$ high-intensity running; TEM $=$ technical error of measurement; $\mathrm{Cl}=$ confidence interval; $n=$ number; $\mathrm{ANOVA}=$ analysis of variance. Significantly different from fullback $(p<0.05)$ and difference above the upper level of TEM 95\% Cl.

Significantly different from forwards $(p<0.05)$ and difference above the upper level of TEM $95 \% \mathrm{Cl}$.

$\S$ Significantly different from hooker $(p<0.05)$ and difference above the upper level of TEM $95 \% \mathrm{Cl}$.

Significantly different from service players $(p<0.05)$ and difference above the upper level of TEM $95 \% \mathrm{Cl}$.

One-way ANOVA significant main effect $(p<0.05)$.

\#Significantly different between first and second halves $(p<0.01$ with pseudo-Bonferroni adjustment).

${ }^{*}$ Significantly different from backs (Bonferroni post hoc; $p<0.05$ ) and difference above the upper level of TEM 95\% Cl.

(Bonferoni post hoc; $p<0.05$ ) and difference above the upper level of $\mathrm{TEM} 95 \% \mathrm{Cl}$. 

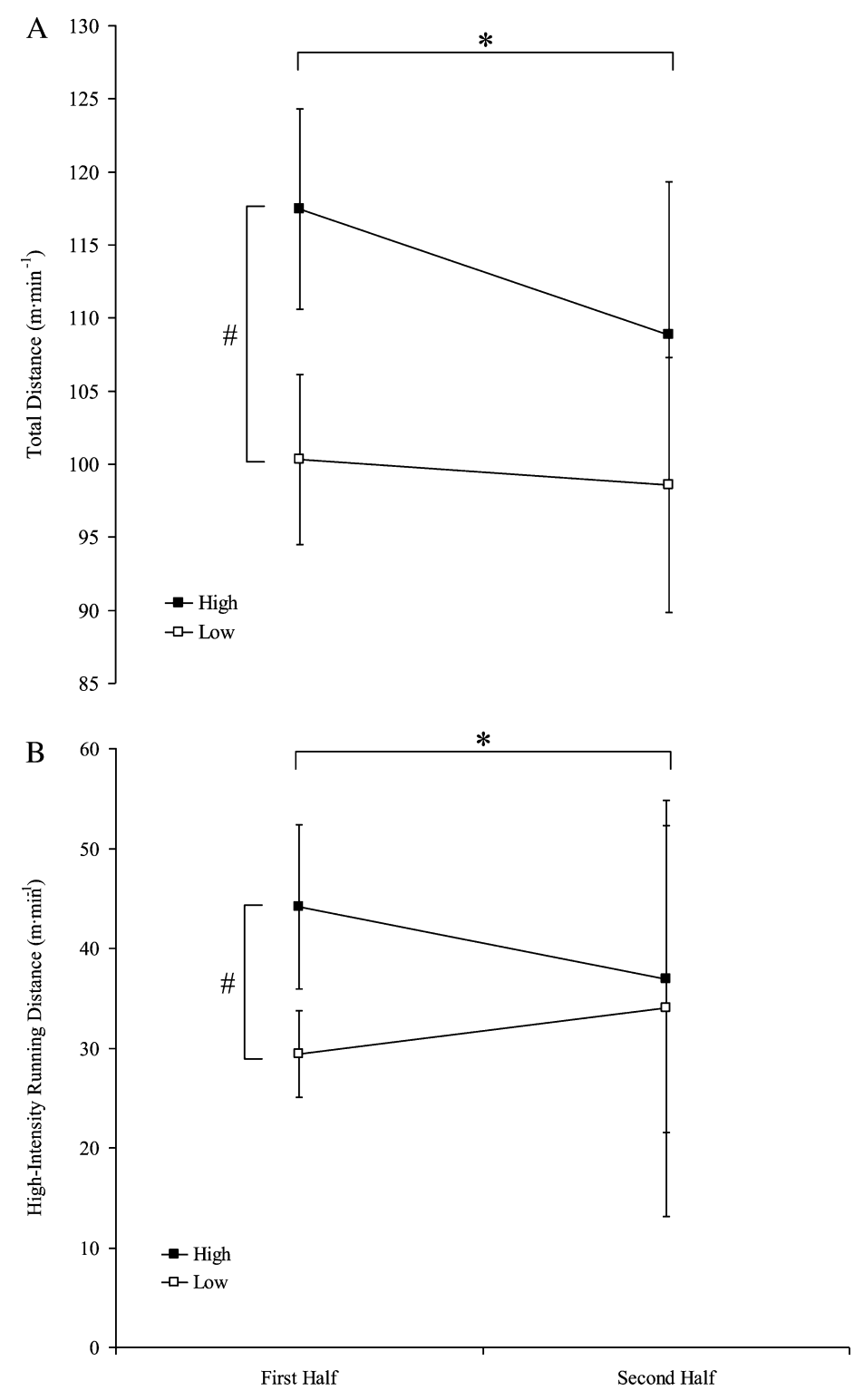

Figure 2. Effect of (A) total work rate and (B) high-intensity work rate during the first half on second-half work-rate measures. A group $\times$ time interaction was found for both physical performance variables $(p<0.05)$. High represents the players that had the greatest total and high-intensity work rate for the first half above the median value of pooled data $(n=19)$; Low represents the players that had lowest total and high-intensity work rate during the first half below the median value of the pooled data $(n=19)$. " Significantly different between high-group and low-group $(p<0.05)$. "Significantly different between first and second halves $(p<0.05)$.

recorded. Tackle loss: The opposing team cleared the play-the-ball area before the defensive player involved in the tackle returned to an on-side position.

Reliability. The reliability of the physical and game-specific skill analysis methods used in this study has been described $(8,21)$. The reproducibility of physical performance measures ranged from good-to-poor (1.0-12.9 percentage of technical error of measurement [TEM\%]). The reliability of game-specific skill measures was good (3.9-4.8 TEM\%). To identify 'real' differences in physical and game-specific skill measures between positional groups, the absolute TEM with $95 \%$ confidence intervals (TEM 95\% CI) were calculated according to previous methods (21).

\section{Statistical Analyses}

Data were tested for normality and homogeneity using the Shapiro-Wilks statistic, and Levene's test for equality in variances, respectively. A oneway between-groups analysis of variance (ANOVA) was used to determine differences in physical performance and game-specific skill measures between the 5 positional groups for the first half, second half and match. If significant main effects were found, Bonferroni post hoc analysis was performed. Differences in physical performance and game-specific skill measures between the first and second halves for the 5 positional groups were determined using Student's paired $t$-test. Because of the use of multiple $t$-tests, a Bonferroni adjustment was applied to each dependent variable group (i.e., time, exercise intensity, distance travelled, frequency, speed, and game-specific skill measures). Thus, statistical significance was set at an alpha level of 0.01 ( $p \leq 0.05 / 5)$. A 2way $2 \times 2$ factorial ANOVA was used to examine the effect of total and HIR intensity of exercise during the first half on subsequent second-half match exercise intensities. The between-subject factor (first-half intensity of exercise) included 2 groups (i.e., high and low group), whereas the within-subject factor (time) included 2 halves (i.e., first and second halves). When a significant interaction was found (i.e., first-half intensity of exercise $\mathrm{x}$ time) Student's paired $t$-test and unpaired $t$-test were applied to determine differences 
between halves and the 2 groups, respectively. Effect sizes (d) were also calculated and values of $0.2,0.5$, and above 0.8 were considered to be small, moderate, and large effects, respectively (1). Relationships between physical performance and game-specific skill measures were determined using Pearson's correlation coefficient. Statistical significance was set at $p<0.05$, and all data are reported as the mean $\pm S D$ unless otherwise stated. Analyses were performed using SPSS (Version 15.0, Chicago, IL).

\section{Results}

\section{Performance Measures}

Table 1 shows the intensity of exercise and mean distance traveled during each movement category for the entire match for the 5 positional groups. Table 2 shows the total playing time and frequency per minute of playing time and mean duration for each movement category performed during a match by each positional group. High-intensity to low-intensity ratio for the match was similar between the backs, forwards, fullback, hooker, and service players (1:9.5, $1: 7.2,1: 7.3,1: 7.6$, and 1:7.9, respectively; $p=0.174)$. Likewise, there was no difference in the mean match playing speed between the backs, forwards, fullback, hooker, and service players $(6.1 \pm 0.4,6.4 \pm 0.6,6.7 \pm 0.6,6.7 \pm 0.6$, and $6.4 \pm$ $0.5 \mathrm{~km} \mathrm{~h}^{-1}$, respectively; $p>0.185$ ). Although there was no difference in total intensity of exercise between any of the positional groups (Table $1 ; p=0.261$ ), the intensity of exercise during backward movement was greater in the fullback than any in other positional groups (Table 1; $p<0.001$ ).

\section{High-Intensity Match Activities}

Figure 1 shows the relative time spent sprinting, VHIR, HIR, and mean time between sprints for the entire match for the 5 positional groups. The fullback performed more VHIR bouts per minute of total playing time than any other positional group (Table 2; $p=0.007$ ). Both the intensity of exercise (Table $1 ; p=0.008$ ) and relative time spent at VHIR $(p=0.026)$ and sprinting $(p=0.006)$ were greater for the fullback than for the hooker and service players. The fullback (149 \pm 63 seconds) had a lower mean recovery time between sprints than the hooker had $(284 \pm 113$ seconds), which was outside the upper limit of TEM $95 \%$ CI (19-37 seconds).

\section{Low-Intensity Match Activities}

The backs spent the most time walking during a match, which was greater than that spent by the forwards, hooker, and service players $(50.7 \pm 4.2$ vs. $41.6 \pm 2.9,39.3 \pm 4.8$, and $41.8 \pm 3.7 \%$, respectively; $p<0.001)$. In addition, the hooker spent a greater amount of time jogging than did the backs and forwards $(29.3 \pm 1.7$ vs. $23.1 \pm 2.3$ and $25.1 \pm 2.8 \%$, respectively; $p<0.001$ ). There were no differences in relative time spent standing for the backs, forwards, fullback, hooker, and service players $(16.2 \pm 5.0,20.5 \pm 5.0,15.5 \pm$ $3.0,18.9 \pm 3.3$, and $19.0 \pm 4.1 \%$, respectively; $p=0.125$ ).

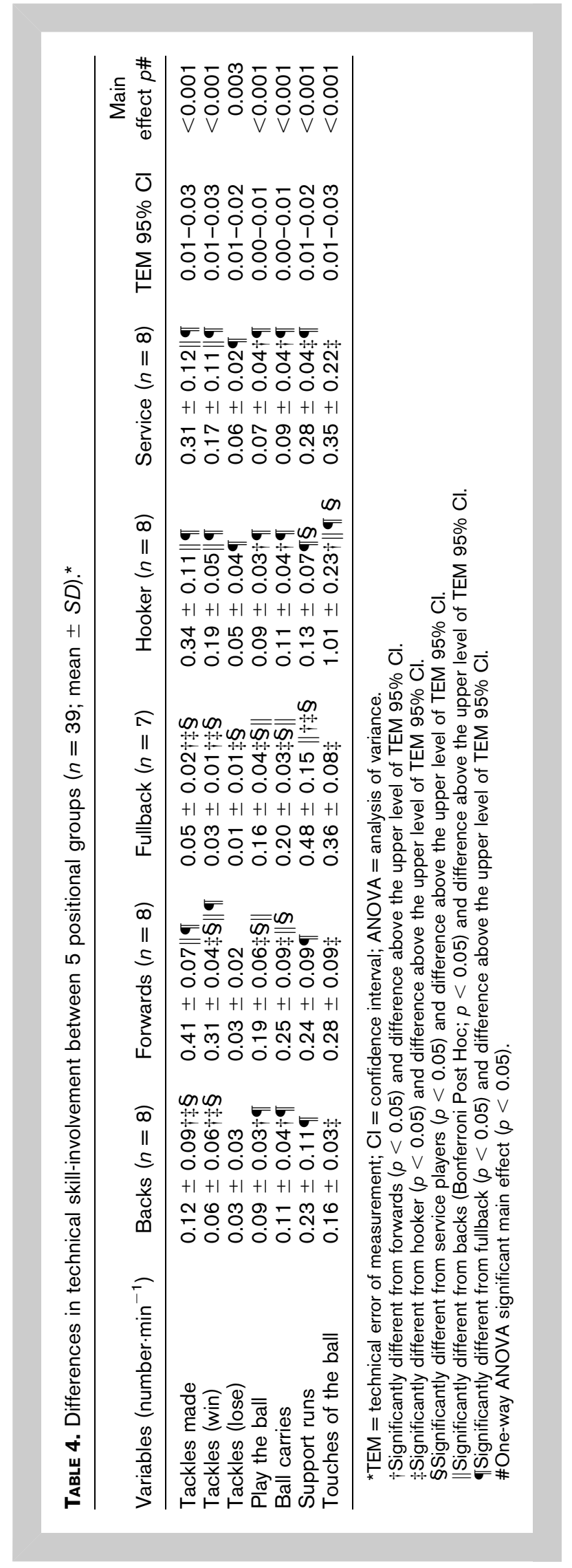

VOLUME 25 | NUMBER 11 | NOVEMBER 2011 | 3083 
When combined, there were no differences in relative time spent in LIR for the match between the backs $(90.1 \pm 2.0 \%)$, forwards $(87.2 \pm 2.5 \%)$, fullback $(87.7 \pm 2.2 \%)$, hooker $(87.5 \pm$ $3.9 \%)$, and service players $(88.4 \pm 2.0 \%, p=0.217)$.

\section{First Half- and Second-Half Activities}

The mean playing time for the first and second halves was $40.2 \pm 9.1$ and $40.2 \pm 9.3$ minutes, respectively. Table 3 shows the intensity of exercise, relative time spent, and frequency of sprinting and VHIR, and changes in speed for the first and second halves between the 5 positional groups. The fullback had a higher intensity for VHIR during the second half than any other positional group (Table $3 ; p=0.004$ ). The fullback also had a higher sprinting intensity during the second half than the forwards, hooker, and service players (Table 3; $p=0.002)$.

\section{Effect of First-Half Activity on the Second Half}

A significant interaction was found between the high- and low-group first-half intensity of exercise $\mathrm{x}$ time (Figure 2). Players who had a greater total intensity of exercise or intensity for HIR for the first half (high group) decreased their total or HIR exercise during the second half (Figure 2; $p<0.05)$. Conversely, players who had a lower total intensity of exercise or intensity for HIR during the first half (low group) did not show a decrease in total or HIR exercise during the second half (Figure $2 ; p>0.05$ ). Effect sizes were very large for total intensity of exercise $(d=1.1)$ and small for HIR exercise $(d=0.2)$.

\section{Game-Specific Skill Measures}

Table 4 shows the game-specific skill involvement per minute of total playing time for the 5 positional groups. The number of tackles made per minute of total playing time was greater for the forwards, hooker, and service players than the backs and fullback (Table $4 ; p<0.001$ ). There was no difference in the range of one-on-one tackles performed during a match for backs $(0-2)$, forwards $(0-2)$, fullback $(0-3)$, hooker $(0-1)$, and service players $(0-4)$, respectively $(p=0.364)$. The hooker touched the ball on more occasions than any other positional group did (Table $4 ; p<0.001$ ). The fullback performed more support runs than did any other positional groups (Table $4 ; p<0.001$ ). A strong relationship was found between the number of supports performed per minute of total playing time by the fullback to intensity for VHIR $(r=0.825)$, VHIR bouts completed per minute $(r=0.802)$ and relative time spent at VHIR $(r=0.840)$ for the match, respectively $(p<0.05, n=7)$. The forwards also showed a moderate relationship between the number of tackles made per minute of playing time with the number of sprints performed per minute during the match $(r=0.746$, $p=0.039, n=8)$.

\section{Discussion}

The purpose of this study was to determine whether differences exist in either physical or game-specific skill demands between positional groups in professional rugby league. The results show that positional roles play an important part in determining the amount of physical and game-specific skill involvement during match play. There is currently little data available on the match-specific demands of professional rugby league, and therefore, these results may have implications for the design of specific physical conditioning drills that may incorporate game-specific skillrelated activities common to each positional role.

The major physical performance differences between playing positions was that the fullback was involved in more very high-intensity activity and had a >twofold higher exercise intensity during backward running for the match compared to all other positions. Although the higher exercise intensity during backward running may be explained by the unique defensive role of the fullback, the greater involvement in very high-intensity activity was mainly because of completing more of this activity during the second half than other positional groups. Important roles of the fullback are to both direct defensive structure, cover the goal line from kicks and breaks in play and to run the ball or support a ball-carrier during attacking play. These roles demand that the fullback is often positioned well behind $(10-30 \mathrm{~m})$ the defensive line, which places more space between the fullback and the opposition players. The fullback is observed to face the opposition when retreating to this location, and this may explain the increased backward running. Moreover, the greater space between the fullback and the opposition may allow the fullback to generate speed and reach higher velocities more often than the other positions. These results may be used by coaches to develop specific training programs for fullbacks. Indeed, such programs may include a greater component of HIR in both the forward and backward direction. It may be that specific small-sided rugby league games can be designed with specific rules that require the fullbacks to regularly position themselves in and out of the defensive line. However, generic interval training and speedagility training programs may also be appropriate for this purpose.

The mean sprint duration and distance data in this study are similar to those reported for several other professional teamsport codes such as rugby union and soccer $(5,16,20)$. In the current investigation, mean sprint distances ranged from $16.0 \mathrm{~m}$ for the fullback to $21.1 \mathrm{~m}$ for the backs with no between position differences. Additionally, maximum sprinting distances or durations rarely exceeded $50 \mathrm{~m}$ or 6 seconds, with players completing $\sim 30$ or more sprints depending on their playing position during a match. These results highlight the intermittent nature of rugby league. Given the brief duration of sprints ( $\sim 2.3$ seconds) across positional groups, acceleration appears to be the most crucial factor in performance for all positions (7). Based on these results, speed development should form an integral part of sprint training. This type of training should focus on sprint distances between 15 and $20 \mathrm{~m}$ for all positional groups, with fullbacks 
being prepared to complete more sprint efforts than the forwards, hooker, or service players. Using sprint distances of $>50 \mathrm{~m}$ during training may be detrimental to the development of acceleration and would reduce specificity to match play.

In agreement with previous research (18), this study shows that the ability to perform physical activity during the second half is related to the physical stress of the first half in professional rugby league. Specifically, players who completed more physical activity in the first half showed a decrement in physical performance during the second half in both total intensity of exercise and intensity for HIR. Conversely, when a lower physical stress was placed on the players during the first half, second-half total intensity of exercise and HIR exercise was maintained. However, the small effect size $(d=0.2)$ observed for the HIR exercise suggests that the effect of HIR performed during the first half on second-half physical performance is only relatively small. This may be because of the larger measurement error associated with high-intensity activities when using the CBT method described in this study. Nevertheless, the results support the previous finding in professional soccer (18) and Australian rules football (2) that second-half match play is related to the fatigue induced by the activities completed in the first half. These results highlight the potential benefit of real-time global positioning systems or alternate matchanalysis systems to aid in player substitution during top-level team sports, including rugby league. Furthermore, these results may also be used to develop specific physical conditioning drills.

Defensive skills are an important element of rugby league match play, often increasing the physical work required, and are perceived by players to be one of the most fatiguing components of the game $(6,13)$. The present results show that the forwards have the highest involvement in defensive skills during match play. The forwards perform the most tackles, often sprinting to make the tackle. This quick defensive-line speed may be linked to the forwards' attempt to reduce the impact force of the attacking player and to minimize advancement in field position. The forwards were also involved in the highest number of tackle wins, consequently increasing the level of physical exertion experienced by this positional group. Although the hooker and service players are also largely involved in tackling during match play, they tend to lose more tackles than the forwards (i.e., $\sim 14-20$ vs. $\sim 7 \%$, respectively). This is most likely because of the smaller physical size (9) and lower absolute strength (17) of these positional groups. Additionally, the present results show that one-on-one tackles are rarely made by any positional group (i.e., average $\sim 2$ per game). This change from previous research (14) could be because of a shift in coaching philosophy, placing higher importance on slowing the play the ball by generating numerous players in each tackle. Although defensive skills may be important for all professional rugby league players, the present results clearly highlight the importance of high level defensive skills for forwards. In particular, forwards may benefit from physical training that improves defensive-line speed. Additionally, it is possible that hookers and service players may be assisted in improving their defensive skills by increasing their physical size and absolute strength.

Offensive skills are also an important determinant of rugby league performance adding to the high-physical workload experienced during match play. The results show that the fullback performs the most offensive skills, primarily because of supporting the ball-carrier on more occasions. Similar to the forwards, the fullback is also heavily involved in running the ball and completes a high number of play the balls, indicating a tendency to hold onto the ball. This is consistent with previous research showing that forwards spend more time taking the ball up and playing the ball than the backs and were less likely to pass the ball to a team member (14). In contrast, the service players rarely run the ball or consequently play the ball. This may be because of the distinct role of the service players to control offensive structure, often requiring them to support the ball-carrier to set up the next play. Moreover, the large number of involvements with the ball by the hooker is mostly because of their unique role as dummy-half, acting as the primary ball distributor during match play (14). Overall, the backs had the lowest involvement in game-specific skills, mainly because of their positioning on the fringes of the field. However, when they did get the ball, they tended to carry it further because of the space created by the players in the middle of the field. Collectively, these results highlight the need for physical conditioning to incorporate game-specific skill involvement that is position specific. Such training methods lend support to the use of skill-based conditioning drills to adequately prepare positional groups for the demands of a game (11). Indeed, skill-based conditioning games with specific rules may be used to encourage players to complete tasks that reflect their positional demands in competition. For example, small-sided rugby league games played on a larger pitch size with fewer players may provide greater space allowing players to run the ball and create more opportunities for offensive skills to be employed. However, it is important that each player's positional and tactical roles be maintained (e.g., players play the role that would be expected during competition) in these games if position-specific skills are to be developed.

It has been suggested that matches played under the limited interchange rule exhibit a lower mean match speed (10). Although direct comparisons with previous studies are not possible because of differences in methodologies among the studies, the suggested decrease in mean match speed was thought to be because of less recovery time between highintensity efforts (10). However, the high-intensity to lowintensity ratios for the forwards (1:7), backs (1:9.5) and hooker $(1: 8)$ in this study are similar to those of previous research investigating the prop (1:7) (15) or forwards (1:6) 
(14), backs (1:8) (14), and hooker (1:10) (15) at the professional level before the rule modification, and for professional level pivots (1:10 and 1:12) props (1:7) and outside back (1:28) players under the limited interchange rule (23). In contrast, the amount of high-intensity activity performed by the forward and hooker positions in this study was 9.5 and $9.3 \%$ higher than high-intensity activity completed by the prop and hooker positions during an entire match under the unlimited interchange rule (15), but less than outside backs (17.0\%), adjustables (15.9\%), and hitup forwards $(16.9 \%)$ in a recent report of professional players competing under the limited interchange rule in the NRL (12). The differences between these studies may be because of different criteria used to classify high and low activities or the player substitution routine employed by the team in either this or other studies. For example, if the interchange routine for forwards and hookers provided long recovery periods between time involved in the play, it may have allowed more high-intensity involvement when re-entering the match. Overall, these results show that the introduction of the limited interchange rule has had a relatively small effect on the global physical demands of most positional roles in professional rugby league. However, it appears that forwards and the hooker may complete more high-intensity activity during match play under the limited interchange rule.

This study provides new knowledge regarding the physical and game-specific skill demands common to specific positional roles in professional rugby league. Results show that the fullback completed more VHIR because of a greater involvement in offensive skills, while the hooker was in control of the ball on most occasions. Defensively, the forwards, hooker, and service players completed more tackles, with the forwards dominating the greatest amount of the tackles. These results show that positional roles play an important part in determining the amount of physical and game-specific skill involvement during match play in professional rugby league.

\section{Practical Applications}

The present results show that positional roles play an important part in determining the amount of physical and game-specific skill involvement during match play in professional rugby league. Although there were some general similarities in the global exercise intensity profiles between the positions in this study, there are some important differences in both physical and skill demands between many of the positions. Coaches can use these findings to assist in the preparation of training programs that will allow players to cope with both the physical and skill demands of their playing position. Although no studies have examined if game-specific training is the optimal approach for preparing high level team-sport athletes, it is logical that some part (not all) of training programs and conditioning practices should reflect the physical and game-specific skill-related activities of their playing position. These new findings can be used to guide this process in professional rugby league. As examples, fullbacks should be prepared to cope with greater amounts of VHIR, whereas forwards should focus on developing the capacity to complete more defensive work when compared to other positional groups. Well-designed sport-specific small-sided games training may be efficient methods for training these capacities in a group. However, coaches should be aware that there may also be some benefit of players training for demands of other positions as occasionally players are required to play in several positions during a season. Future studies should examine how coaches can effectively replicate these demands in training and if match-specific training is an optimal training strategy for professional rugby league players.

\section{ACKNOWLEDGMenTs}

We would like to thank the rugby league players and Parramatta Eels NRL Club for their assistance in the data collection. We would also like to thank Ermanno Rampinini and Matthew Cameron for their valuable suggestions on the research design and data interpretation. No source of funding was used in preparation for this manuscript.

\section{REFERENCES}

1. Cohen, J. Statistical Power Analysis for the Behavioral Sciences. Hillsdale, NJ: Lawrence Erlbaum Associates, 1988.

2. Coutts, AJ, Quinn, J, Hocking, J, Castagna, C, and Rampinini, E. Match running performance in elite Australian Rules Football. J Sci Med Sport 13: 543-548, 2010.

3. Coutts, AJ and Reaburn, PRJ. Time and motion analysis of the AFL field umpire. J Sci Med Sport 3: 132-139, 2000.

4. Dawson, B, Hopkinson, R, Appleby, B, Stewart, G, and Roberts, C. Player movement patterns and game activities in the Australian Football League. J Sci Med Sport 7: 278-291, 2004.

5. Deutsch, MU, Kearney, GA, and Rehrer, NJ. Time-motion analysis of professional rugby union players during match-play. J Sports $S c i$ 25: 461-472, 2007

6. Docherty, D, Wenger, HA, and Neary, P. Time-motion analysis related to the physical demands of rugby. J Hum Mov Stud 11: 49-52, 1985.

7. Duthie, G, Pyne, DB, and Hooper, SL. Time motion analysis of 2001 and 2002 super 12 rugby. J Sports Sci 23: 523-530, 2005.

8. Edgecomb, SJ and Norton, KI. Comparison of global positioning and computer-based tracking systems for measuring player movement distance during Australian football. J Sci Med Sport 9: 25-32, 2006.

9. Gabbett, TJ. Physiological characteristics of junior and senior rugby league players. Br J Sports Med 36: 332-339, 2002.

10. Gabbett, TJ. Influence of the limited interchange rule on injury rates in sub-elite Rugby League players. J Sci Med Sport 8: 111-115, 2005.

11. Gabbett, TJ. Skill-based conditioning games as an alternative to traditional conditioning for rugby league players. J Strength Cond Res 20: 309-315, 2006

12. King, T, Jenkins, D, and Gabbett, T. A time-motion analysis of professional rugby league match-play. J Sports Sci 27: 213-219, 2009.

13. Larder, P. The Rugby League Coaching Manual. London, United Kingdom: Kingswood Press, 1992.

14. Meir, R, Arthur, D, and Forrest, M. Time and motion analysis of professional rugby league: A case study. Strength Cond Coach 3: 24-29, 1993.

15. Meir, R, Colla, $\mathrm{P}$, and Milligan, C. Impact of the 10 -metre rule change on professional rugby league: Implications for training. Strength Cond J 23: 42-46, 2001. 
16. Mohr, M, Krustrup, P, and Bangsbo, J. Match performance of highstandard soccer players with special reference to development of fatigue. J Sports Sci 21: 519-528, 2003.

17. O'Connor, D. Physiological characteristics of professional rugby league players. Strength Cond Coach 4: 21-26, 1996.

18. Rampinini, E, Coutts, AJ, Castagna, C, Sassi, R, and Impellizzeri, FM. Variation in top level soccer match performance. Int J Sports Med 28: 1018-1024, 2007.

19. Rampinini, E, Impellizzeri, FM, Castagna, C, Coutts, AJ, and Wisløff, U. Technical performance during soccer matches of the Italian Serie A league: Effect of fatigue and competitive level. $J \mathrm{Sci}$ Med Sport 12: 227-233, 2009.
20. Reilly, T and Thomas, V. A motion analysis of workrate in different positional roles in professional football match-play. J Hum Mov Stud 2: 87-97, 1976.

21. Sirotic, AC, Coutts, AJ, Knowles, H, and Catterick, C. A comparison of match demands between elite and semi-elite rugby league competition. J Sports Sci 27: 203-211, 2009.

22. Spencer, M, Lawrence, S, Rechichi, C, Bishop, D, Dawson, B, and Goodman, C. Time-motion analysis of elite field hockey, with special reference to repeated-sprint activity. J Sports Sci 22: 843-850, 2004.

23. Sykes, D, Twist, C, Hall, S, Nicholas, C, and Lamb, K. Semiautomated time-motion analysis of senior elite rugby league. Int $J$ Perform Anal Sport 9: 47-59, 2009. 